\title{
PROKLA-Redaktion
}

\section{Editorial: Imperialistische Globalisierung}

Spätestens angesichts der Kriege im Kosovo, in Afghanistan und im Irak zeigt sich, dass die Welt nach dem Ende des Ost-West-Konflikts keineswegs friedlicher geworden ist. Mit dem Zusammenbruch der Sowjetunion hat zwar die Blockkonfrontation ein Ende gefunden, nicht jedoch die Konkurrenz der Staaten untereinander. Diese findet heute freilich unter den Bedingungen einer neuen strategischen Situation statt: An die Stelle des Gleichgewichts zwischen zwei Supermächten ist nun die einzig verbliebene Supermacht USA getreten, die zunehmend unilateral agiert. Dabei begegnet diese Supermacht völlig neuartigen Koalitionen. Dass, wie zuletzt beim Irakkrieg, die USA mit ihrem Hauptverbündeten England auf die gemeinsame Opposition von Frankreich, Deutschland, Russland und China stoßen, ist ein Novum. Zwar zeichnet sich damit noch keine neue Lagerbildung $a b$, doch ist deutlich geworden, dass die bisherigen weltpolitischen Koordinaten in Bewegung geraten sind. Kein Wunder, dass in dieser Situation auch eine Veränderung derjenigen Konzepte stattfindet, in denen das internationale Staatensystem analysiert wird. Während zu Beginn der 1990er Jahre von verschiedenen Seiten über den tatsächlichen oder vermeintlichen Verlust von nationalstaatlichen Handlungspotentialen diskutiert wurde, wird seit einiger Zeit wieder verstärkt über Imperialismus und Staatenkonkurrenz geredet.

\section{Imperialismustheorien, kapitalistische Entwicklung und Globalisierung}

Unter Imperialismus versteht man gemeinhin, dass sich ein Staat unter Einsatz von militärischen, politischen und ökonomischen Mitteln Einfluss und Vorteile auf der internationalen Ebene verschafft. In den klassischen marxistischen Imperialismustheorien erhielt der Begriff jedoch noch eine spezifische Bedeutung: Lenin, Luxemburg u.a. versuchten zu zeigen, dass bestimmte Probleme der Kapitalverwertung, die in einer fortgeschrittenen Phase kapitalistischer Entwicklung unausweichlich seien, die Staaten zu einer imperialistischen Politik zwingen würden. Eine imperialistische Konkurrenz der am höchsten entwickelten kapitalistischen Staaten wurde daher als notwendiges und "letztes" Stadium kapitalistischer Entwicklung behauptet. Die ökonomietheoretischen Grundlagen dieser These sind aber gerade aus dem Blickwinkel der marxschen Kritik der politischen Ökonomie äußerst brüchig (vgl. etwa zu Lenins Imperialismustheorie die grundlegende Kritik von Christel Neusüß, Imperialismus und Weltmarktbewegung des Kapitals, Erlangen 1972).

Entgegen den Erwartungen der klassischen Imperialismustheorien hat sich die Arena der Weltpolitik seit dem 1. Weltkrieg jedoch mehrmals grundlegend gewandelt. Spätestens nach dem 2. Weltkrieg wurde das internationale Staatensystem nicht mehr durch die Konkurrenz der kapitalistischen Metropolen unter- 
einander dominiert, sondern durch den Ost-West-Konflikt. Innerhalb dieses bipolaren Systems vermochten die USA ihre Hegemonie auszubauen und zu festigen. Erst in den 1970er Jahren geriet diese Hegemonie in eine Krise. Die 1980er und 1990er Jahre waren dann durch einen beschleunigten Globalisierungsschub geprägt und mit dem Zusammenbruch des Realsozialismus entstanden auch in Osteuropa genuin kapitalistische Produktionsverhältnisse. Selbst China, das einzig verbliebene, große realsozialistische Land versucht mittlerweile den politisch kontrollierten Übergang zum Kapitalismus (vgl. PROKLA 119 Chinesischer Kapitalismus, Juni 2000).

Die Globalisierung veränderte und begrenzte die Handlungsmöglichkeiten der Nationalstaaten, wenn auch in höchst unterschiedlichem Ausmaß, für die vielen kleinen und die wenigen großen Länder. Das weltpolitische Szenario lässt sich nicht mehr, wie in den klassischen Imperialismustheorien, auf die Konkurrenz imperialistischer Mächte um Anlagesphären für ihr überschüssiges Kapital reduzieren. Gerade die USA haben sich in den letzten Jahrzehnten zum weltweit mit Abstand größten Kapitalimporteur entwickelt. Zinsen und Wechselkurse, der Ölpreis (da Öl nach wie vor der mit Abstand wichtigste Energieträger ist) und die Währung, die als Weltgeld fungiert, sowie die Regularien des internationalen Handels sind heute die entscheidenden strategischen Größen der Weltwirtschaft, die auch (im Unterschied zu den Löhnen) weitgehend auf globaler Ebene bestimmt werden.

Wird heute über Imperialismus geredet, so kann nicht bruchlos an die klassischen Imperialismustheorien angeschlossen werden. Problematisch sind nicht nur Unterstellungen wie eine das Wertgesetz aushebelnde Monopolisierung oder die Annahme einer Unterkonsumtionstheorie, sondern auch der mehr oder weniger offene Geschichtsdeterminismus der älteren Theorien. Statt den Versuch zu machen, alte Imperialismuskonzepte auf neue Verhältnisse zu übertragen, muss es um Imperialismus innerhalb der Globalisierung gehen, um die verschiedenen Facetten einer „imperialistischen Globalisierung".

Was Imperialismuskonzepte für die Analyse gegenwärtiger Verhältnisse beitragen können, wird in diesem Heft unter ganz verschiedenen Blickwinkeln von WolfDieter Nart und Reinhart Kößler ausgelotet. Während Kößler die ältere Imperialismusdiskussion mit den Globalisierungsdebatten der letzten Jahre vergleicht, bezieht sich Narr vor allem auf die Imperialismusdiskussion der 1960er und 1970 er Jahre und entwickelt das Konzept des ,introvertierten Imperialismus", der kein zu eroberndes Außen, sondern nur ein stets tiefer zu durchdringendes Innen kennt.

\section{Global Governance oder neue imperialistische Konkurrenz?}

Nach dem Ende des Ost-West Konflikts sah es zu Beginn der 1990er Jahre für einen kurzen Augenblick so aus, als ergäbe sich die Möglichkeit einer "Global Governance": einer kooperativen Bearbeitung der großen ökologischen und sozialen Probleme, an der sich staatliche und zivilgesellschaftliche Akteure gleichermaBen beteiligen würden. Nicht Idealismus, sondern die schiere Größe dieser Probleme und die bereits hergestellte weltweite Interdependenz, so die optimistische These, würde auch großen Staaten keine andere Chance lassen, als an diesem Prozess teilzunehmen (vgl. zur Auseinandersetzung um Global Governance die Beiträge von Achim Brunnengräber/Christian Stock in PROKLA 116, September 1999, sowie Dirk Messner und Franz Nuscheler in PROKLA 118, März 2000). Die 1990 er Jahre erwiesen sich jedoch als Jahrzehnt der Krisen, Kriege und verstärkter staatlicher Konkurrenz. Zu Beginn des neuen Jahrtausends toben weltweit 47 Kriege und bewaffnete Konflikte. Die Finanz- und Währungskrisen in Asien, Lateinamerika, Osteuropa und der Tür- 
kei haben für Millionen von Menschen eine massive Verschlechterung ihrer alltäglichen Lebensbedingungen gebracht. Effektive Maßnahmen, um hier gegenzusteuern, sind nicht in Sicht. Der Abbau sozialer Sicherungssysteme in den meisten der entwickelten kapitalistischen Länder schreitet rasant voran - um den "Standort“ für das internationale Kapital attraktiv zu halten. Und in Verbindung mit dem „Kampf gegen den Terrorismus" nimmt auch der Ausbau staatlicher Kontroll- und Repressionsmaßnahmen erneut zu.

Allerdings ist in den letzten Jahren auch eine immer breitere Protestwelle entstanden, die sich aus unterschiedlichen Motiven und von verschiedenen Positionen aus den Zumutungen des globalen Kapitalismus entgegen stellt. Weltweit haben sich in den 1990er Jahren soziale und politische Gruppen in einer bis dato einzigartigen Art und Weise vernetzt. Die mittlerweile jährlich stattfindenden regionalen und globalen Sozialforen bieten Globalisierungskritikern, Antirassismusinitiativen, stadtpolitischen Gruppen, Umweltschützern und vielen anderen mehr die Möglichkeit zum Austausch und geben ihnen eine globale Öffentlichkeit. Kein offizielles Gipfeltreffen von Seattle über München bis Davos konnte in den vergangenen Jahren stattfinden, ohne dass es durch massive Proteste von Gruppen verschiedenster Couleur gestört worden wäre. Als die USA Anfang 2003 in den Irak einmarschierten, protestierten am 15. Februar weit über 10 Millionen Kriegsgegner gleichzeitig in Tokyo, New York, Rom, Berlin und anderen Städten rund um die Welt. Nach wie vor kann den USA an politischem Einfluss und militärischer Stärke kein anderes Land auch nur das Wasser reichen, allerdings ist ihre ökonomische Stellung längst nicht mehr so unangefochten wie ehedem. Gemessen an Marktgröße, Wirtschaftskraft und Produktivität ist die EU dabei, mit den USA gleichzuzichen. Entscheidender ökonomischer Vorteil der USA bleibt die Rolle des Dollar als Weltgeld, ein Vorteil, den die USA allerdings auch bitter nötig haben: ermöglicht doch vor allem die besondere Rolle des Dollar, dass das „Twin Deficit" (bestehend aus einem Haushaltsund einem Leistungsbilanzdefizit) bisher problemlos durch internationales Kapital finanziert wurde, das in die USA floss. Genau diese Rolle des Dollar, und damit ein wichtiger ökonomischer Stützpfeiler der US-Hegemonie, wurde aber durch die Einführung des Euro attackiert. Zwar hat der Euro noch längst nicht die Bedeutung des Dollar als Reserve-, Handelsoder Anlagewährung erreicht, allerdings ist mit dem Euro zum ersten $\mathrm{Mal}$ seit dem 2. Weltkrieg überhaupt ein Kandidat in Sicht, welcher dem Dollar die Rolle als Weltgeld streitig machen könnte, und in den letzten Jahren hat die weltweite Bedeutung des Euro durchaus zugenommen. Insofern lässt sich das zunehmend unilaterale Agieren der USA in den letzten Jahren und insbesondere der Irakkrieg auch als Versuch des politischen und ökonomischen Terraingewinns gegenüber potenziellen Konkurrenten verstehen, wo es neben der Kontrolle von Ölproduktion, Öltransport, Ölpreis auch um die Kontrolle der Währung, in welcher die Ölrechnungen zu bezahlen sind, ging - was im übrigen genau den Zielen entspricht, die im Frühjahr 2001 im Cheney-Report (benannt nach dem US-amerikanischen Vizepräsidenten) formuliert wurden. $\mathrm{Ob}$ sich dies alles auch so durchsetzen lässt, ist allerdings eine andere Frage.

Um mit den USA tatsächlich um die Rolle als Hegemon zu konkurrieren, müsste die EU aber nicht nur auf ökonomischer, sondern auch auf militärischer Ebene eigenständig Stärke demonstrieren, was mit erheblichen Kosten und voraussichtlich auch innen- wie auBenpolitischen Konflikten verbunden wäre. Immerhin treiben gerade Deutschland und Frankreich, die im Vorfeld des Irakkrieges offensiv gegen die USInterventionspolitik aufgetreten sind und sich damit als „Friedensmächte ${ }^{6 \sigma}$ profi- 
lierten, die von den USA unabhängige Militarisierung der EU engagiert voran. Bereits 1999 hat die EU auf dem Gipfel von Helsinki die Einrichtung einer eigenen, weltweit operierenden, europäischen Eingreiftruppe beschlossen. Eine Stärke von 60.000 Soldaten ist angepeilt. Der unmittelbar vor seiner Annahme stehende EU-Verfassungsentwurf gießt die Militarisicrung der EU in Recht und Gesetz. So heißt es etwa im Artikel 40 Absatz 1, dass die ,gemeinsame Sicherheits- und Verteidigungspolitik integraler Bestandteil der Gemeinsamen Außen- und Sicherheitspolitik ist. Sie sichert der Union die auf zivile und militärische Mittel gestützte Fähigkeit zu Operationen.“ Und in Absatz 3 dieses Artikels zwingt die EU ihre Mitgliedstaaten faktisch zur militärischen Aufrüstung: „Die Mitgliedstaaten verpflichten sich, ihre militärischen Fähigkeiten schrittweise zu verbessern." Und insbesondere in Deutschland gehört es zu einer der wichtigsten Kontinuitäten von schwarz-gelber zu rot-grüner Politik, dass Auslandseinsätze der Bundeswehr zum akzeptierten Normalfall werden.

\section{Globale Anarchie nach dem Ende des Multilateralismus?}

Offensichtlich stehen wir in einer Umbruchsituation, ohne dass bereits klar erkennbar wäre, in welche Richtung die künftige Entwicklung geht. Auf jeden Fall scheinen sich multilaterale Ansätze der Weltpolitik, wie sie sich in den frühen 1990er Jahren abzeichneten, eher aufzulösen als zu konsolidieren. Was dies auf der Ebene des Welthandels bedeutet, untersucht Heribert Dieter in seinem Beitrag. Inzwischen wird die 1995 gegründete Welthandelsorganisation WTO, die in der Vergangenheit vor allem we- gen ihrer strikten Liberalisierungspolitik kritisiert wurde, zunehmend von den USA aber auch von der EU durch bilaterale Vereinbarungen umgangen, $\mathrm{da}$ sie so ihre Interessen noch besser durchsetzen können. In Europa stieß vor allem der seit dem Amtsantritt von Bush junior verstärkte Unilateralismus auf Kritik. Dass sich Unilateralismus und Multilateralismus aber keineswegs dichotom gegenüberstehen, zeigt der Beitrag von Christoph Görg und Markus Wissen. Ausgehend von Poulantzas' Staatstheorie zeigen sie am Beispiel der Auseinandersetzung um das TRIPS-Abkommen über geistiges Eigentum, wie Uni- und Multilateralismus ineinander verschränkt sind. Von entscheidender Bedeutung für die weitere Entwicklung des Weltsystems dürfte das künftige Verhältnis zwischen den USA und der EU sein. An diesem Punkt gehen denn auch die Meinungen am weitesten auseinander. Während Ingo Schmidt zwar eine Reihe ökonomischer Differenzen, aber letztlich doch weitgehend übereinstimmende Herrschaftsinteressen diesseits und jenseits des Atlantiks ausmacht, sieht Immanuel Wallerstein den weiteren Niedergang der USA als eine weitgehend ausgemachte Sache an Damit verbunden wären aber nicht nur stärker werdende Differenzen zwischen den USA und der EU, sondern das Entstehen einer ,globalen Anarchie", in der sich auch ganz neue Machtzentren herausbilden könnten, etwa durch eine Annäherung von China, Japan und Korea. Die Diskussion ist also alles andere als abgeschlossen. Wir werden sie daher auch in den nächsten Heften, außerhalb des jeweiligen Heftschwerpunktes fortsetzen. 


\section{In eigener Sache}

Am 4. Oktober fand in Berlin die Mitgliederversammlung der „Vereinigung zur Kritik der politischen Ökonomie e.V." statt. Die Vereinigung gibt die PROKLA heraus, diskutiert die künftigen Schwerpunktsetzungen und wählt die Redaktion. Neu in die Redaktion gewählt wurden Alex Demirovic, Henrik Lebuhn und Gregor Zattler; die übrigen Redaktionsmitglieder wurden wiedergewählt. Birgit Mahnkopf hat nach 18jähriger außerordentlich engagierter und produktiver Mitarbeit leider nicht mehr kandidiert. Die Redaktion dankt ihr für ihren Einsatz, ihre Diskussionsfreude und ihre vielen anregenden Ideen. Der PROKLA bleibt Birgit Mahnkopf aber auch weiterhin als 2. Vorsitzende der "Vereinigung" verbunden.

Im Vordergrund der Debatten der Mitgliederversammlung stand in diesem Jahr die gefährdete ökonomische Situation der PROKLA. Wie andere linke politischwissenschaftliche Zeitschriften hat auch die PROKLA mittlerweile mit sinkenden Verkaufszahlen und wachsenden ökonomischen Problemen zu kämpfen. Dabei liegt der Rückgang der Verkäufe nicht daran, dass die Abonnentenzahl geringer würde, sondern vor allem am abnehmenden Einzelverkauf; die Buchhandlungen verringern zunehmend ihr Sortiment und halten ältere Titel oder auch Zeitschriften wie die PROKLA überhaupt nicht mehr vorrätig.

Die PROKLA benötigt Unterstützung. Neue Abonnements würden helfen, aber auch mehr Präsenz in Buchhandlungen und Bibliotheken. Wer von unseren Lesern und Leserinnen die Möglichkeit hat, neue Abonnenten zu werben, möge dies bitte tun. Wer die PROKLA weitergehend unterstützen möchte, ist herzlich eingeladen Mitglied in der „Vereinigung zur Kritik der politischen Ökonomie e.V.“ zu werden. Mit den Mitgliedsbeiträgen unterstützt die Vereinigung die Finanzierung der Redaktionsarbeit. Monatliche oder jährliche Mitgliedsbeiträge können nach eigenem Ermessen bezahlt werden und sind steuerabzugsfähig, da die Vereinigung als gemeinnützige Körperschaft anerkannt ist.

Die PROKLA lädt nicht nur zur Einsendung von Artikeln bzw. Exposes ein, wir freuen uns auch über Vorschläge für künftige Themenschwerpunkte. Ebenso erwünscht sind kritische Beiträge zu bereits publizierten Artikeln oder Themen. Neben dem jeweiligen Heftschwerpunkt sollen in Zukunft auch verstärkt Debatten über aktuelle Themen geführt werden. Dabei sollten Debattenbeiträge mit ca. 30.000 Zeichen (1012 Druckseiten) in der Regel etwas kürzer sein als die üblichen Artikel des Heftschwerpunkts mit ca. 40-55.000 Zeichen (14-20 Druckseiten). 